\title{
URSS: OPTIMISMO POR EL ESPACIO EN EL DISEÑO DE LOS AÑOS 60
}

\author{
USSR: Space Optimism in the 1960s' Design
}

\author{
Renata CAnevari, Sofía Venzel \\ Universidade de Vigo, España
}

\begin{tabular}{ll} 
KEY WORDS & ABSTRACT \\
\cline { 2 - 3 } $\begin{array}{l}\text { Design } \\
\text { Space Age }\end{array}$ & This paper is based on a review of historical events in the USSR, which \\
Optimism & took place against the backdrop of the golden decade of the Soviet space \\
Society & age - the 1960 - and its relationship to the institutionalization of design \\
USSR & in the country. The Soviet space design, little mentioned in the prevailing \\
& discourses of the discipline's history, was a vehicle for the propaganda of \\
& socialism and holds interesting facts about social pride and optimism for \\
the space. The interpretation of everyday artifacts from that Soviet \\
period reaffirms the rhetorical power of design and consists of a \\
meaningful representation of its material and visual culture.
\end{tabular}

PALABRAS CLAVE

Diseño

Era espacial

Optimismo

Sociedad

URSS

\section{RESUMEN}

Este trabajo parte de una revisión de acontecimientos históricos ocurridos en la URSS, que tuvieron como telón de fondo la década de oro de la era espacial soviética -los años 60- y su relación con la institucionalización del diseño en el país. El diseño soviético espacial, poco mencionado en los discursos predominantes de la historia de esta disciplina, fue un vehículo de propaganda del socialismo y encierra interesantes datos acerca del orgullo y optimismo de la sociedad por el cosmos. La interpretación de artefactos de uso cotidiano de ese periodo soviético reafirma el poder de retórica del diseño y es una representación significativa de su cultura material y visual.

Recibido: 05/05/2020

Aceptado: 26/06/2020

\section{GLOBAL KNOWLEDGE}




\section{Introducción}

$\mathrm{E}$ l 'telón de acero'1, entre la URSS -aliada a otros países del bloque socialista- y la Europa Occidental, impidió que se conociera parte de la historia de la Unión Soviética. No obstante, una de las conquistas soviéticas que cambió el curso de la humanidad, es de todos conocida: el lanzamiento del primer satélite artificial, el Sputnik I (PS-1), en 1957. Su forma esférica, con cuatro antenas, fue el primer símbolo del éxito de las misiones espaciales soviéticas, que se reflejó en el diseño de los bienes de consumo de ese país.

En la segunda década de la Guerra Fría ${ }^{2}$, mientras Occidente se recuperaba del "Shock Sputnik", poco se sabía acerca de cómo vivía la sociedad soviética esos momentos del otro lado del telón. Según investigaciones ${ }^{3}$ realizadas, la conquista del espacio se vivió con orgullo y entusiasmo social en los sesenta, que se plasmaron en varios ámbitos culturales, entre ellos, el diseño.

La temática del cosmos dejó claro en esa década el poder de retórica del diseño que impregnado de dimensiones simbólicas- también fue un potente canal mediante el cual la propaganda del Partido Comunista intentaba legitimar el éxito del régimen y elevar la moral de la población respecto al socialismo.

En este trabajo se intenta explorar el vínculo entre el diseño soviético como cultura material y visual y algunos mitos creados alrededor de la era espacial a lo largo de los años 60 (cuando su práctica se convirtió en una realidad en el país): desde la gloria hasta los duros golpes al optimismo por el cosmos, causados por una serie

\footnotetext{
1 Iron Curtain (Telón de Acero) fue un término acuñado a principios de los años 1940s por Sir Winston Churchill para referirse a la frontera física e ideológica que existía entre los países del bloque oriental respecto al resto del mundo occidental (www.britannica.com, "iron curtain”, párr. 1)

${ }^{2}$ La Guerra Fría, fue el periodo de tensiones políticas, sociales y económicas entre los bloques de países capitalistas y socialistas, liderados respectivamente, por E.E.U.U. y la U.R.S.S. que empezó después de la Segunda Guerra Mundial (1946) (Véase Powaski, 2000: 91-93).

3 Véase Soviet Space Mythologies: Public Images, Private Memories, and the Making of a Cultural Identity de Slava Gerovitch (2015), Soviet Space Culture: Cosmic Enthusiasm in Socialist Societies, de Maurer, E., Richers, J., Rüthers, M. y Scheide, C. (Eds.) (2011), Made in Russia: Unsung Icons of Soviet Design de Michael Idov (2011).
}

de sucesos internos y por la llegada del hombre estadounidense a la Luna (1969).

Para esa exploración, se analizarán algunos ejemplos de diseños con motivos espaciales que formaban parte del repertorio visual y material de esa sociedad, y que tienen un importante vínculo con la cultura popular de los sesenta, y aún a día de hoy se mantienen como símbolos de la identidad cultural rusa.

Este estudio tiene por objeto difundir algunos momentos de la historia del diseño global - a través de relatos menos conocidos de esa disciplina en los años 60. Además, es un intento de hacer hincapié en su poder de retórica y su capacidad para convencer a los ciudadanos, y pretende demostrar que el componente alegórico del diseño soviético de esa década tuvo funciones más allá de lo estético, que cambiaban de significado y provocaban distintos sentimientos, según evolucionaba la sociedad.

Se ha señalado 4 que en la historia del diseño prevalece un discurso occidental, que se centra en la cronología de sucesos que ocurrieron en países anglosajones e industrializados, lo que contribuye a que ésta se retroalimente de las mismas versiones. Por esta razón, algunos de los aspectos que justifican este trabajo, son: la inclusión de narrativas alternativas a la historia de la disciplina, así como la generación de discusiones sobre si puede existir el diseño en una economía planificada. Además de ello, la totalidad de las referencias encontradas sobre el diseño cósmico soviético de esa década, están en ruso o inglés, por lo que una parte sustancial de este trabajo se ha basado en las traducciones de Sofía Venzel, que contribuyen a la generación de un corpus de conocimiento en español sobre esa materia poco estudiada.

Para la realización de este estudio, se revisó documentación técnica y no técnica, documentales rusos $\mathrm{y}$, además, se recogieron datos en dos entrevistas a personajes clave, sobre la temática tratada aquí: Aleksandra Sankova, directora del Museo del Diseño de Moscú y comisaria de la exposición "Diseño soviético de 1950 a 1989" y Anastasia Voronina, del Museo Estatal de Historia de los Urales del

\footnotetext{
${ }^{4}$ Autores como Jonathan Woodham, Tony Fry y Victor Margolin hacen esa afirmación en sus estudios.
} 
Sur y comisaria de la exposición "Crónicas del diseño soviético".

Se afirma que, para analizar el diseño soviético de ese periodo, es imprescindible contextualizarlo dentro de las singulares circunstancias políticas, económicas y sociales en las que se encontraba el país en la posguerra y en el período posestalinista. Así, una parte significativa del estudio está dedicada a ese escenario, que posibilitó la eclosión del diseño en el país. La apertura política del 'deshielo', los planes sociopolíticos de Nikita Khrushchev, los consecutivos éxitos del programa espacial soviético, la creciente insatisfacción de los ciudadanos por la escasez de bienes de consumo $y$, finalmente, la decepción de los ciudadanos por el proyecto fallido de socialismo utópico, que llegó a finales de los sesenta y que, veinte años más tarde culminaría en el colapso de la URSS, enmarcan el periodo estudiado.

Aunque ese período de la URSS ejerza fascinación en los historiadores, la cronología de su diseño ha sido poco estudiada y sólo empezó a difundirse en la última década, a través de exposiciones ${ }^{5}$, libros $^{6}$ y documentales ${ }^{7}$. Debido a la dificultad de encontrar documentación perdida o destruida tras la disolución de la Unión Soviética, incluso en Rusia, su historia sólo ha empezado a salir a la luz de forma sistemática a partir de 2012, con la inauguración del Museo del Diseño de Moscú 8: el primer museo íntegramente dedicado a recuperar la memoria del diseño soviético. Por ello, se propone tener en cuenta la presencia de la historia del diseño en países como la URSS, con una realidad política y económica distinta a la capitalista, con la que suele estar asociada.

\footnotetext{
5 “Soviet Design 1950-1980", 2012. Comisariada por: Alexandra Sankova y Alyona Sokolnikova, Organizada por: Moscow Design Museum

${ }^{6}$ Designed in the USSR (2016), VNIITE - Discovering Utopia: Lost Archives of Soviet Design (2018) y Made in Russia: Unsung Icons of Soviet Design (2011)

7 VNIITE. Discovering Utopia (2016).

Producido por: Andrey Silvestrov y Alexandra Sankova

Con guión de: Lusiya Artemieva y Olga Druzhinina

Edición y Traducción: Sveta Chirkova

${ }^{8}$ En 2012, se fundó en Moscú, el Moscow Design Museum, que reunió documentos y objetos del periodo soviético, además de realizar entrevistas a diseñadores de la época.
}

\section{Comienzos de la Era Espacial soviética, la carrera espacial y su relación con el Diseño}

La llamada Era Espacial soviética empezó de forma repentina (Devezas et al., 2012: 963) con el lanzamiento del primer satélite artificial, Sputnik I, en 1957. Ese "bip" que emitió el satélite, mientras orbitaba alrededor de la Tierra, sacudió al mundo occidental y provocó el "Shock Sputnik", que suscitó la idea de que los ingenieros y diseñadores occidentales no tenían la misma creatividad e ingenio que los soviéticos (Idov, 2011: 14-16) (Bayazit, 2004: 18) (Cropley, 2016: 155) (Rüthers, 2011: 207). La relación entre cohetes y tecnología armamentista, así como el pasado de conflictos bélicos entre oriente y occidente, hizo que ese satélite llevara el mensaje implícito de la superioridad del socialismo (Maurer, Richers, Rüthers, Scheide, 2011: 3).

Devezas et al sugieren que los viajes espaciales soviéticos causaron temor y suscitaron profundos debates políticos en Estados Unidos (2012: 967). En consecuencia, el gobierno estadounidense empezó a dedicar grandes recursos a la investigación de las actividades proyectuales y creativas (i.e. diseño e ingeniería) (Bayazit, 2004: 18). Al año siguiente del lanzamiento del Sputnik, el entonces presidente de Estados Unidos, Eisenhower (1890-1969), firmó la Ley Nacional del Espacio y la Aeronáutica (1958), para la creación de la NASA (National Aeronautics and Space Administration) (Idov, 2011: 16), dando comienzo así a la carrera espacial.

La delantera de la URSS en esa carrera, llegó en un delicado momento político, en el que el Partido Comunista, liderado por Nikita Khrushchev, buscaba relanzar la ideología socialista para las nuevas generaciones (Maurer, Richers, Rüthers y Scheide, 2011: 4). Sputnik y, posteriormente, Gagarin, parecían ser las imágenes ideales para ayudar al Estado en ese cometido (Rüthers, 2011: 206).

En aquel momento, el diseño, la ingeniería, la tecnología y las ciencias se convertían en símbolos de la supremacía de la URSS y contaban con el entusiasmo popular. Un discurso político pautado en ellos y orientado al futuro, tenía en la conquista del espacio grandes posibilidades de recuperar la confianza de los ciudadanos en el 
sistema socialista, debilitado por la escasez de recursos y suministros a la población (Siddiqi, 2011: 283). El diseño entraba en la ecuación de los elementos necesarios para convencer a los ciudadanos sobre las ventajas del socialismo: diseño, bienes y cosmos.

En la URSS de Stalin, los bienes de consumo que no fueran estrictamente necesarios, no se consideraban. Como afirma Azrikan, "los productos industriales se utilizaban sólo para construir el socialismo" (1999: 45). Millones de ciudadanos llevaban un estilo de vida austero y no contemplaban una vida de consumo, hasta que empezaron a comparar su realidad con la de otros países, tras la llegada de los soldados victoriosos de los frentes de la II Guerra Mundial en trenes, en los que traían consigo productos occidentales de Alemania, Austria y Europa Oriental (Idem: Ibídem). Tras la muerte del dictador, la comparación entre el estilo de vida soviético y el del resto del mundo, se intensificó con la política de apertura del país hacia occidente.

\section{El 'deshielo' y el estado de los bienes soviéticos}

El periodo de apertura de la URSS, llamado 'deshielo' (thaw), empezó con la llegada del primer secretario del Partido Comunista, Nikita Khrushchev (1894-1971) al poder, tras la muerte de Iósif Stalin (1878-1953) y dio lugar a un sentimiento de libertad y crítica social (Maurer, Richers, Rüthers y Scheide, 2011: 4). Se empezó a criticar abiertamente la mala calidad "de los bienes soviéticos en comparación con los occidentales", debido a las pésimas condiciones de la industria y a su "falta de unidad estilística" (Karpova, 2013: 628-631). Esto dejó clara la urgencia en mejorar la vida cotidiana de los ciudadanos a través del diseño.

El proceso de "desestalinización" generó tensiones sociales que se convirtieron en un gran desafío político para el Kremlin. Las principales, según afirma Rüthers, fueron la brecha entre "las promesas y la realidad respecto al abastecimiento de insumos", además de "los costes elevados de la carrera armamentista y las imágenes de la cultura occidental de consumo" (2011: 206). En la segunda mitad de los 1950s, según Karpova, "el entorno material adquiría cada vez más peso político" (2013: 631) y a partir de ahí, los dirigentes soviéticos empezaron a entender el "poder diplomático de la Guerra Fría" (Idem: Ibidem) y permitieron que el "telón de acero" se abriera al mundo occidental con algunas iniciativas de intercambio cultural $y$ político entre las naciones. Esa apertura a otras culturas y regímenes, hizo que los soviéticos, ya insatisfechos por la falta de bienes, se hicieran más conscientes y todavía más difíciles de convencer por el régimen.

\section{American National Exhibition - 1959. Estilo de vida soviético vs. American Way of Life}

Debido a uno de esos convenios de intercambio cultural y político, dos millones de soviéticos, visitaron la exposición American National Exhibition, instalada en el parque Sokolniki (Moscú -1959), sobre el estilo de vida capitalista estadounidense, con ejemplos de coches, refrescos e incluso una réplica de una casa típica de las afueras de sus ciudades, completamente amueblada con lo mejor del diseño del momento (A. Sankova, videoconferencia, 23 de febrero de 2019) (Idov, 2011: 6-7) (Choate, 2010: 94).

En la cocina de esa exposición, se produjo el célebre Kitchen Debate (Debate de la Cocina). El entonces vicepresidente Richard Nixon presumía del "American Way of Life"9 (McGuirk in Moscow Design Museum, 2018) ante Khrushchev. Sobre todo, de las comodidades de su sistema, de las que los ciudadanos estadounidenses podían disfrutar. En un intento de no quedarse atrás y aprovechar la amplia difusión del evento en los medios para promocionar su política (Choate, 2010: 94-95), Khrushchev, afirmó que la URSS estaba a la altura de los EEUU:

\section{"[Khrushchev y Nixon entran en la cocina de la Exposición Americana]}

Nixon: Quiero enseñarle esa cocina... [Nixon señala el lavavajillas] Khrushchev: Nosotros tenemos cosas de ese tipo.

\footnotetext{
${ }^{9}$ Expresión sobre el estilo de vida estadounidense que se fundamenta en la idea que se tiene sobre las libertades y derechos individuales de sus ciudadanos, así como en sus oportunidades de prosperar económicamente en ese país.
} 
Nixon: Puede que en algunos temas ustedes estén por delante de nosotros... pero en otros puede que lo estemos nosotros-en televisión en color, por ejemplo.

Khrushchev: No, también estamos a su altura en eso (...) Los estadounidenses han creado su propia idea del hombre soviético. Pero no somos como ustedes creen, que los soviéticos se quedarán boquiabiertos al ver esta exposición, pero la realidad es que las nuevas viviendas soviéticas tienen ahora todos esos aparatos.

Nixon: Sí, pero... Khrushchev: En la Unión Soviética, para tener una casa, todo lo que usted tiene que hacer es haber nacido allí. Se tiene el derecho a una vivienda... En EE.UU., si Vd. no tiene un dólar, tiene el derecho de dormir en una casa o en el suelo. A pesar de ello, dicen Vds. que somos esclavos del comunismo.

Nixon: Reconozco que usted es muy elocuente y enérgico...

Khrushchev: Enérgico no es lo mismo que razonable" (Transcripción "Debate de la Cocina", "www.cia.gov", 24/07/1959)

Khrushchev alardeó del éxito de los cohetes y satélites del programa espacial soviético (Idov: 2013: 7), pero se dio cuenta de que los electrodomésticos y otros bienes de esa casa, alimentaban la fantasía popular de la URSS sobre la cultura estadounidense de masas (Rüthers, 2011: 216).

Tras ese acontecimiento, se hizo evidente "la importancia del consumo doméstico como factor del poder político" (Karpova: 2013: 631). Khrushchev y el Partido comprendieron que las carencias de la industria y la poca calidad de los bienes de consumo podrían minar la fe del pueblo en el sistema, y empezaron a tomar medidas. Su plan político (Plan de Siete Años, 1959-1965) prometía mejoras en el día a día de los ciudadanos (Rüthers, 2011: 213). Entre las iniciativas, se encontraba suplir la escasez de bienes de consumo en los hogares, a través del incentivo a la "industria ligera, y en la producción de los muy demandados bienes de consumo, olvidados en los programas políticos anteriores" (Maurer, Richers, Rüthers y Scheide, 2011: 4). A partir de ahí, el gobierno se centraría más en atender a las necesidades materiales de los ciudadanos.

\section{La paradoja: bienes de consumo y viajes espaciales como moneda de cambio para fortalecer el socialismo}

En 1957, Khrushchev había lanzado una campaña de viviendas colectivas; una iniciativa social, política y económica que prometía dar un piso a cada familia soviética (Karpova, 2013: 631), cuando lo que predominaba entonces eran los pisos comunales (kommunalka) o barracones compartidos entre varias familias. Gradualmente, millones ${ }^{10}$ de ciudadanos se trasladaron a los apartamentos proyectados (Idem: 632) conocidos como Khrushchevkas- de reducidas dimensiones (entre 22 a $30 \mathrm{~m}^{2}$ ) (Choate, 2010: 61). Eso provocó la demanda de diseño y fabricación de muebles y bienes de consumo adaptados a esas características (A. Sankova, videoconferencia, 23 de febrero de 2019).

En la década de sesenta, las políticas oficiales de relanzamiento del régimen seguían prometiendo brindar al pueblo más bienes de consumo, pero además les ofrecía un grandioso proyecto de viajes espaciales, a cambio de colaborar en la construcción del nuevo socialismo (Rüthers, 2011: 213). Los ciudadanos más conscientes con el 'deshielo', ya no admitían volver al estilo de vida anterior $\mathrm{y}$, aunque las misiones espaciales generaron optimismo, también evidenciaron que, cuanto más se invirtiese en el espacio, menos dinero se destinaría a sus necesidades en la Tierra (Idem: 206 y 215).

No obstante, a pesar de las tensiones sociales, los consecutivos éxitos del programa espacial soviético hicieron que fuera inevitable que el sentimiento de orgullo creciera en la nación. El lanzamiento del Sputnik, el vuelo de los primeros seres vivos al espacio, entre otros, sostenían la propaganda y fortalecían las esperanzas de la población en el futuro; pero el culmen de ese sentimiento llegó con el primer viaje espacial tripulado por un hombre: Yuri Gagarin, que se convirtió en el gran icono del éxito soviético y héroe de todos los tiempos. Gerovitch sostiene que esos éxitos repercutieron en la generación de la posguerra que, "por primera vez, sentía que

\footnotetext{
10 Choate afirma que entre los años 1956 y 1970 tuvo lugar una campaña masiva de viviendas, en la que más de 126 millones de ciudadanos pudieron trasladarse a esos 34 millones nuevos pisos (2010: 56).
} 
ese triunfo significaba la mayor recompensa tras años de sacrificio durante la guerra y las privaciones de la época de Stalin" (2015: 48).

El viaje de Gagarin al espacio, el 12 de abril de 1961, impresionó a todos. Khrushchev se aseguró de que la ceremonia fuera pública y multitudinaria, pero no se imaginó una reacción tan emotiva de los ciudadanos. A tal punto, que las autoridades tuvieron trabajo en contener a las masas por las calles de Moscú (Gerovitch, 2015: 128-129). Una exciudadana soviética recuerda que la alegría de la población fue tal, que -los rusos, que suelen ser fríos en sus demostraciones de afecto- se abrazaban en las calles ese día (Burkova y Digovtsev, 2016). La galardonada con el Premio Nobel de Literatura, Svetlana Aleksiévich, menciona en su libro ${ }^{11}$, cómo ese hito histórico influenció su educación con respecto al régimen: "Mi padre solía recordar que su fe en el comunismo surgió a raíz del vuelo de Yuri Gagarín ‘Hemos sido los primeros! ¡Somos capaces de todo!', y en esa fe nos educaron él y mamá" (Aleksiévich, 2015: II). Ese acontecimiento se consideró dos años después en una encuesta realizada a los jóvenes soviéticos como "el mayor logro humano del siglo" (Gerovitch, 2015: 48).

Ese fuerte sentimiento de entusiasmo vinculado al cosmos, fue utilizado por el Partido y trasladado a los bienes de consumo. La sonrisa de Gagarin estaba presente en una gran variedad de objetos de uso diario, y representaba "todo lo bueno" del socialismo (Idem: 162). Los cosmonautas estaban presentes en todas partes como "nuevos héroes del socialismo": medios de comunicación, sellos y murales, entre otros (Rüthers, 2011: 206), lo que afianzó el papel del diseño cósmico como canal propagandístico del régimen.

\section{El 'cosmos' y el diseño en la agenda de las nuevas políticas estatales}

En octubre de 1961 se puso en la agenda del $22^{\circ}$ Congreso del Partido Comunista, la creación del "Nuevo Hombre Soviético", un plan que utilizaría a los cosmonautas como modelo de ciudadano a seguir: valiente e intrépido (Gerovitch, 2015: 48). Se elegían para el programa espacial, los cosmonautas (hombres y mujeres) de rasgos

\footnotetext{
${ }^{11}$ Véase “El Fin del Homo Sovieticus” (Aléksievich, 2015).
}

eslavos que se identificaran con el pueblo y promovieran los valores comunistas ("www.smithsonianmag.com", 2019, párr. 35). Todo formaba parte de una gran Propaganda.

En ese mismo congreso, el diseño empezó a formar parte de la política estatal, fruto de la demanda por el aumento y mejora de bienes y su repercusión en la política (Woodham, 2004: 446) (Voronina, 2019) (Sankova y Druzhinina, 2018: 13). El 28 de abril de 1962 el Consejo de Ministros aprobó un nuevo programa, que tenía por objeto mejorar la calidad de los productos industriales y los bienes de consumo, y se elaboró un proyecto del Decreto № 394 "Sobre Maquinaria Industrial y Mejora de la Calidad de los Bienes de Consumo a través de la Implementación Metodológica de la Ingeniería Artística" (Azrikan, 1999: 47) (Idov, 2013: 7) (Sankova in Moscow Design Museum, 2018: 8-9) (Karpova, 2013: 636). En el decreto se establecía un sistema educativo de diseño en el país a partir del cual se crearon departamentos de "Ingeniería Artística” en las escuelas de arte, politécnicos y universidades (Azrikan, 1999: 49) (Karpova, 2013: 636). "Ingeniería Artística", fue el extraño término ideado para denominar el "Diseño", pero según Azrikan, para que el decreto fuera aprobado por las autoridades, el término diseño -que recordaba las "amenazas" del capitalismono podría aparecer (1999: 48) (Karpova, 2013: 637). Y, en efecto, no aparecería hasta el año 198612, ya en los años de la 'perestroika'13.

El tema central de ese decreto fue la creación del VNIITE (1962-2014): Instituto Federativo de Investigación Científica Técnico Estética y de sus oficinas de diseño (Azrikan, 1999: 49), que incluía una sede central en Moscú y diez filiales en las principales ciudades de toda URSS (Sankova in Moscow Design Museum, 2018: 8). Ese Instituto, un capítulo poco conocido en la historia del diseño mundial, fue la mayor organización individual de diseño del mundo

\footnotetext{
12 La palabra "diseño" (design) apareció en 1986 por primera vez en un folleto que publicitaba el congreso de la Sociedad de Diseñadores de la URSS. La sociedad fundada gracias a los esfuerzos de Yuri Soloviev (exdirector de la VNIITE), hizo posible que el término se legitimara en el idioma ruso (Véase Designed in the USSR, 2018: 161).

13 La Perestroika (reestructuración en ruso) fue un plan de transición del régimen de economía planificada y centralizada hacia las primeras democratizaciones empresariales en el país durante el gobierno de Mikhail Gorbachev (N. del autor).
} 
(Sinclair in Sankova y Druzhinina, 2018: 4), y probablemente la mayor iniciativa de un Estado sobre políticas de diseño de la historia.

Con la dirección de Yuri Borisovich Soloviev, el instituto contaba con una red de colaboradores de varios ámbitos profesionales ${ }^{14}$ (Azrikan, 1999: 50), incluidos millares de diseñadores 15 : los "ingenieros-artísticos", responsables de la presentación visual de sus productos (Sankova in Moscow Design Museum, 2018: 8). El equipo, formado por pensadores y talentos artísticos, encontró en el VNIITE, más que una oportunidad de mejorar el entorno material: allí se desarrolló "un contexto teórico y práctico de un sistema completo de diseño" (Idem: Ibidem). Esos profesionales intentaron crear la cara humana de la convivencia entre sociedad y su relación con el entorno, a través de proyectos que pensaban más en las personas y en la construcción de una nueva sociedad, que en la construcción del socialismo (Karpova, 2013: 641). En departamentos como Ergonomía, Historia y Diseño para el Futuro- Investigación y Desarrollo (Azrikan, 1999), quedaba claro el compromiso de desarrollar un diseño de calidad pensado para el usuario.

Sin embargo, a pesar de sus intenciones de mejora social a través del diseño, los bienes de producción tenían baja prioridad para el Partido, que tenía preferencia por las necesidades de la industria pesada y militar (Woodham, 2004: 446). La contradicción existente entre la innovación de los diseñadores y las limitaciones e intereses de fabricación -que procuraban unificar y abaratar costes-, hicieron que la mayoría de los proyectos del VNIITE no pasaran del papel (Azrikan, 1999: 68) (Karpova, 2016: 241) (Idov, 2013: 7-8).

Pese a las dificultades para producir objetos originales, el legado de esos "artistas-técnicos" o "ingenieros-artísticos" se consideró una verdadera 'declaración de intenciones'. Dmitri Azrikan, ex-diseñador de la VNIITE, afirma que

\footnotetext{
${ }^{14}$ Ingenieros, representantes de las artes aplicadas, investigadores del arte, críticos, historiadores, psicólogos, filósofos, arquitectos, artistas gráficos, modelistas, entre otros (Azrikan, 1999: 50)

15 En los primeros años de institución, la VNIITE, empleaba a más de 3000 profesionales. Las oficinas de diseño, vinculadas a las industrias principales, tenían alrededor de doscientos grupos de entre 2 y 15 diseñadores que trabajaban en fábricas independientes. Véase $A$ Dictionary of Modern Design (Woodham, 2004)
}

esos profesionales eran conscientes de que sus trabajos nunca serían realidad, pero, aún así, proponían una realidad mejor que la que se vivía en aquella época; una utopía a través de sus proyectos (Azrikan apud Idov, 2013: 8).

Una parte significativa del repertorio visual y material soviético de los años 60 salió de esa institución (Sankova in Moscow Design Museum, 2018). Anastasia Voronina, comisaria de la exposición "Crónicas del diseño soviético"16, afirma que es evidente que en el diseño de esa década se presta especial atención a la temática espacial (A. Voronina, correo electrónico, 15 de mayo de 2019) y aunque algunos de los diseños de aparatos de uso doméstico o personal, eran imitaciones de diseños producidos en occidente (Idov, 2013: 8), otros muchos fueron diseñados y producidos en la URSS, fruto de ese entusiasmo por el cosmos.

\section{La cultura popular y la era espacial}

La principal inspiración de la cultura popular soviética de los sesenta parecía ser la era cósmica, cuyo espíritu se reflejó en la música, el cine, la moda, el diseño, entre otros. En los hogares soviéticos se podría encontrar "casi cualquier objeto doméstico diseñado para incorporar de alguna forma la idea de viajes intergalácticos" (Idov, 2013: 88). La omnipresente influencia del espacio en el entorno visual y material en la vida de los ciudadanos, quedó retratada de manera anecdótica en el documental ruso "Moda Espacial" (Мода на космос):

"Te despiertas por la mañana y ves un cartel con Yuri Gagarin en el espacio, te preparas un té y en la taza también ves temas espaciales, estrellas y Sputnik. Pones la radio y el coro de jóvenes comunistas está cantando una canción sobre el espacio. Miras por la ventana y ves un mural pintado en el edificio de enfrente con un motivo espacial. No importa que sólo unas pocas personas pudieran ir al espacio, porque el espacio estaba por todas partes.

La mítica aspiradora Raketa (Cohete) y los gorros llamados Escafandra, los muebles con formas aerodinámicas y los adornos del árbol de la fiesta de fin de año con forma de

\footnotetext{
16 Exposición organizada por el Museo Estatal de Historia de los Urales del Sur, en 2017.
} 
astronauta. El espacio está por todas partes, en la arquitectura, en postales... películas $\mathrm{y}$ dibujos animados sobre el espacio" (Burkova y Digovtsev, 2016).

Esos estímulos casi subliminales acompañaban a los ciudadanos, tanto en los medios de comunicación masivos, como en los espacios públicos y privados.

\section{Iconos del Cosmos}

La cronología de las misiones espaciales está reflejada en el diseño de finales de los 1950s y toda la década de sesenta, $\mathrm{y}$, como afirma Idov, sus iconos están representados en una larga lista de bienes de consumo. Desde el diseño industrial al gráfico, las formas esféricas y aerodinámicas de las naves, planetas o cohetes, inundaban hogares y oficinas; La temática futurista y las caras de los cosmonautas -humanos o animales- estaban en paquetes de tabaco, caramelos, chocolatinas, postales, entre otros (Idov, 2013: 16 y 88).

Entre los diversos símbolos espaciales presentes en la cultura soviética, se presentan algunos que posiblemente sean los principales iconos del éxito del programa espacial de la URSS y su relación con el diseño de la época:

\section{Sputnik I}

El primer satélite artificial (1957), se llamaba Prosteishii Sputnik, que en ruso significa "satélite elemental". Su nombre (elemental) guarda relación con su diseño sencillo, que consistía en una esfera con cuatro antenas. El diseño de Sergey Pavlovich Korolyov ${ }^{17}$ (1907-1966), formó parte de la memoria de una generación. El satélite simbolizó el poder de recuperación económica del país tras la guerra, cuando los soviéticos anunciaron el inicio de la era cósmica (Maurer, Richers, Rüthers y Scheide, 2011) e inspiró el diseño de diversos bienes. Los "soportes de taza [podstakannik], sellos, postales, llaveros, pisapapeles (...) De pronto, todos los aspectos del diseño industrial empezaron a absorber sus curvas" que reflejaron el espíritu de la era espacial (Idov, 2013: 14-16).

\footnotetext{
${ }^{17}$ Encontrado también con otra grafía: 'Sergei Korolev'.
}

Figura 1. Cartel Propaganda "Nos propusimos una meta para crear una producción industrial socialista Y la hemos alcanzado"

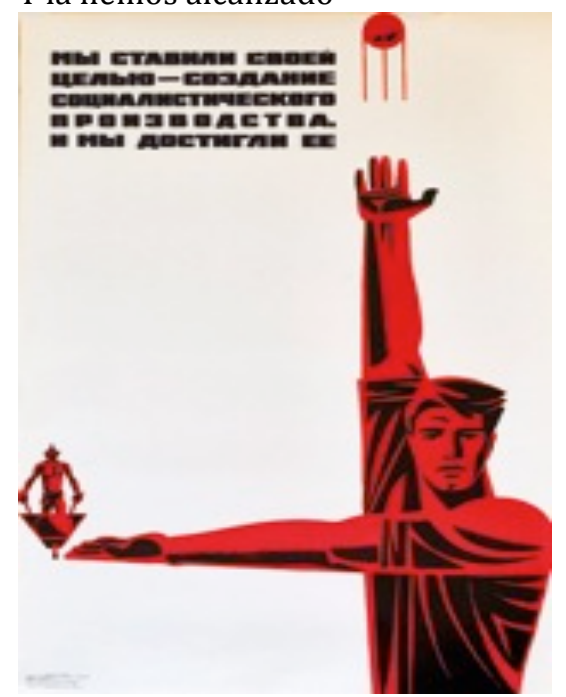

Fuente: Museo del Diseño de Moscú.

Figura 2. Réplica del satélite original

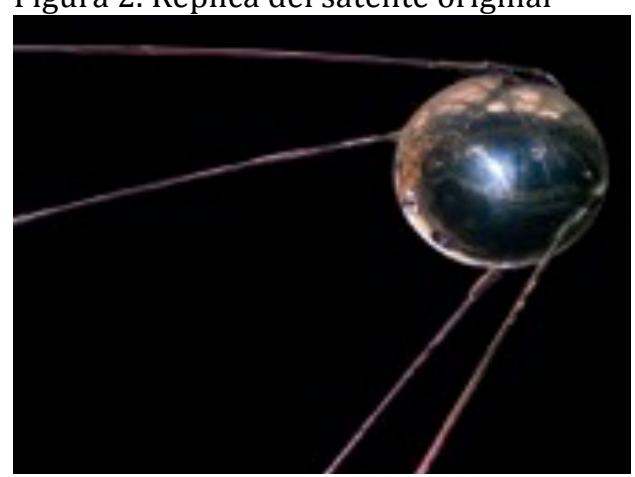

Fuente: Museo Estatal de Historia de los Urales del Sur.

Figura 3. Caja de máquina de afeitar Sputnik. Fabricado por: Leningrad Patefon Factory, 1968

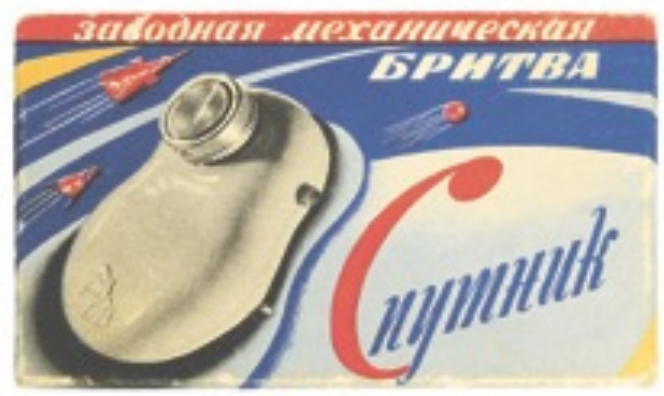

Fuente: Museo del Diseño de Moscú.

\section{Yuri Gagarin}

El primer hombre en volar al espacio era un completo desconocido del pueblo soviético hasta que regresó del espacio. Sus rasgos eslavos y el 
hecho de ser hijo de agricultores, fueron el ejemplo del "Nuevo Hombre Soviético". En abril de 1961, su vuelo espacial se tornó el nuevo símbolo del progreso soviético hacia un futuro brillante y, desde entonces, el cosmos dominó los medios soviéticos y la propaganda del estado (Rüthers, 2011: 207). Yuri es considerado el héroe soviético de todos los tiempos. Su rostro ilustró innumerables objetos: carteles, sellos postales, carátulas de discos, entre otros.

Figura 4. Poster "El cuento de hadas se convirtió en realidad". Diseño de: Boris Staris. Publicado por: Molodaya Gvardia (1961)

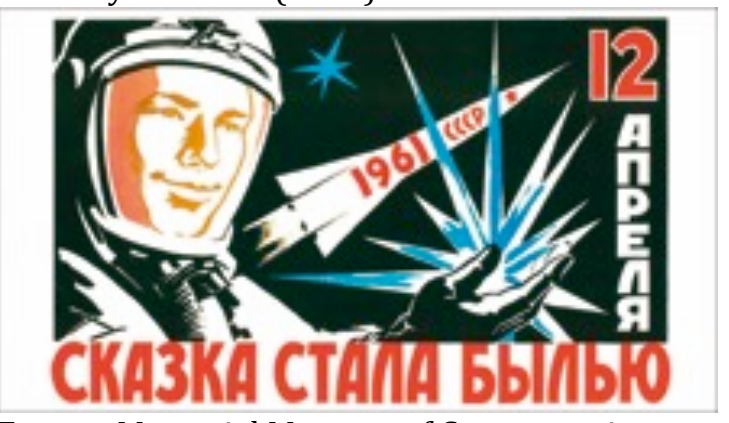

Fuente: Memorial Museum of Cosmonautics.

Figura 5. Grabación de la voz de Yuri Gagarin para la revista musical Krugozor (1960s)

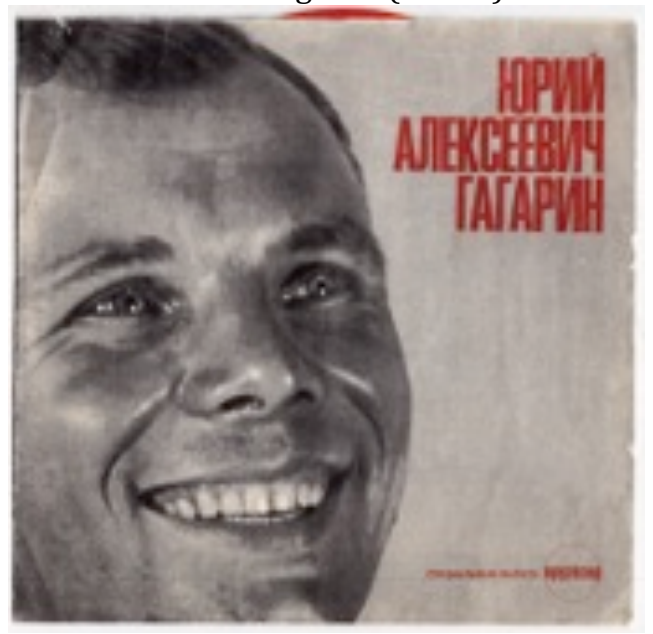

Fuente: Museo del Diseño de Moscú.

\section{Laika, Belka y Strelka}

Poco tiempo después del lanzamiento del Sputnik, la primera perrita espacial, Laika, despegó rumbo al infinito para celebrar el $40^{\circ}$ aniversario de la Gran Revolución de Octubre ${ }^{18}$, aunque no se había contemplado que sobreviviera al vuelo. Belka y Strelka (Ardilla y Flechita) fueron los primeros animales en volver con vida del espacio en agosto de 1960 (Moscow Design Museum, 2018). Sus hocicos estaban presentes en todas partes: Laika, Belka y Strelka aparecen en envoltorios de caramelos, cajas de chocolate, en sobres, sellos, postales, paquetes de cigarrillos, además de estar presentes en muchos juguetes y otras figuritas de souvenir (Ruthers: 2011: 207) (Burkova y Digovtsev, 2016). Las perritas se convirtieron en heroínas y se conservan disecadas en el Museo de la Cosmonáutica de Moscú hasta hoy.

Figura 6. Caja de chocolate de papel y cartón "Belka y Strelka. Viajeras espaciales" (1960s). Fabricado por: "Krasny Oktyabr Fábrica de Chocolate" (Turkina, 2014: 180)

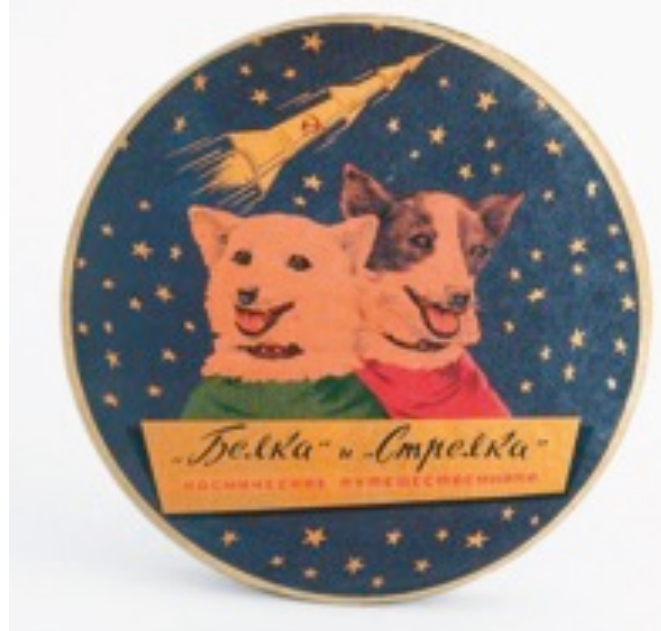

Fuente: Museo del Diseño de Moscú.

Valentina Tereshkova - La primera mujer en viajar al espacio (1963), con tan sólo 26 años, fue la única mujer en emprender ese viaje sola. La hazaña de la cosmonauta, así como su pasado en una fábrica textil, hizo que se convirtiera en un icono de la moda. Su peinado, labios rojos, accesorios y los colores favoritos de sus prendas, fueron la marca de su estilo. Pero quizás su mayor legado fue poner sobre el tapete los debates sobre la igualdad de género, al comentar que los diseños del programa espacial estaban pensados 'por hombres y para hombres' ("anothermag.com", 2015) (Maurer, Richers, Rüthers y Scheide, 2011: 7).

\footnotetext{
${ }^{18}$ Revolución Rusa de 1917.
} 
Figura 7. Sello conmemorativo (1965). "La primera mujer astronauta del mundo. Valentina Tereshkova”. (Fabricado: URSS, 19/07/1963)

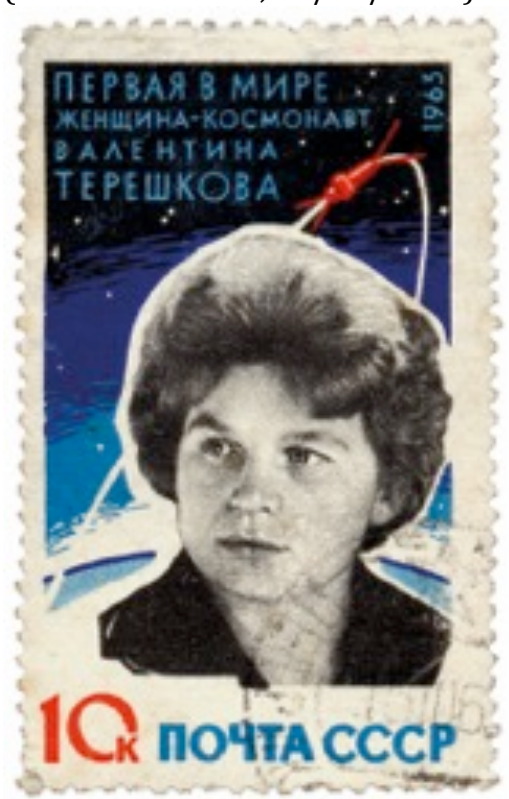

Fuente: http://astronaut.ru/philo/text/010.htm

\section{Diseños que reflejan el optimismo por el espacio en la cultura material y visual soviética}

La temática espacial se manifestó en el diseño gráfico, editorial y de producto, entre otros. Las aspiradoras son ejemplos icónicos de esa exaltación por el espacio. Esos electrodomésticos fueron pioneros en ello y, para Idov, la expresión máxima de la era espacial, reflejada en utensilios domésticos (2013: 88-90). Con colores vivos, formas redondeadas y líneas suaves, se asemejaban a cohetes, planetas o satélites. Incluso algunos nombres hacían referencia a la temática. Los más conocidos, se llamaban Raketa (Cohete), Chaika (Gaviota) y Saturnas (Saturno). Aunque algunos ya habían sido producidos a finales de los años cincuenta, pertenecieron a la cultura material de los sesenta y posterior, ya que su calidad y durabilidad hicieron que permanecieran muchos años en los hogares soviéticos.
Figura 8 y 8.1. Saturnas (1962). Fábrica de maquinaria: Welding Lugar: República Socialista Soviética de Lituania
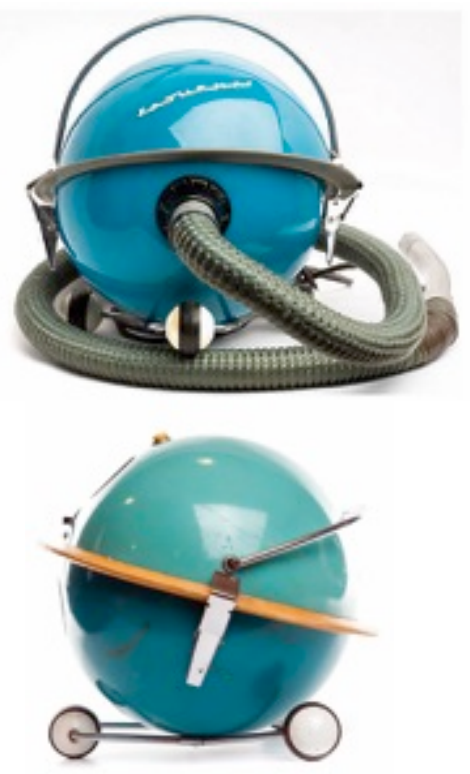

Fuente: Museo Estatal de Historia de los Urales del Sur.

Figura 9. Chaika “Gaviota” (1963). Aspiradora universal. Planta de fabricación de maquinaria Kommunar

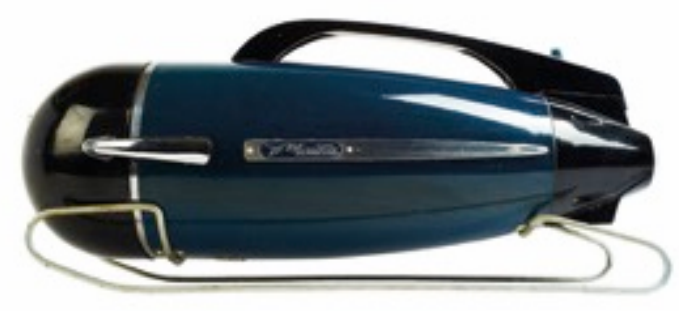

Fuente: Museo del Diseño de Moscú.

Los relojes con diseños inspirados en el espacio fueron un "ejemplo de la calidad del trabajo de los ingenieros-artísticos" (diseñadores) de esa década. Aquí se ven dos ejemplos emblemáticos de Molniya, un fabricante de relojes para vehículos de guerra y cohetes espaciales, que proyectó modelos conmemorativos tras el éxito de las misiones espaciales. El modelo Molniya cuyo nombre significa "Rayo, relámpago", que en ruso es sinónimo de velocidad, rapidez, ambición, aspiración, competición, es muy simbólico en los años de la carrera espacial y de la Guerra Fría. Vostok que significa "este" en ruso, también es el nombre de la cápsula en la que viajó Yuri Gagarin. Es todo un clásico del diseño soviético y fue aclamado en el mercado mundial. En él puede 
apreciarse la historia de la exploración del cosmos por el hombre, desde el lanzamiento (este) hasta el vuelo alrededor de la Tierra (A. Voronina, correo electrónico, 15 de mayo de 2019).

Figura 10. Molniya "Rayo" (1966). Reloj de mesa con luz. de cobre y cristal. Colores: negro y dorado. Fabricante de relojes Molniya. Lugar: Chelyabinsk.

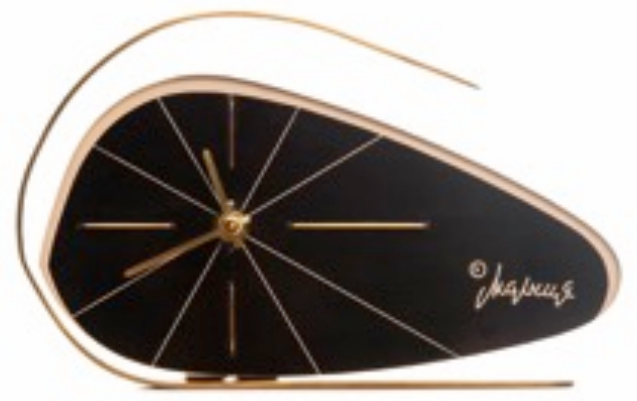

Fuente: Museo Estatal de Historia de los Urales del Sur.

Figura 11. Vostok 1 "El Este” (1961). Reloj de mesa. Fabricante de relojes: Molniya. Lugar: Chelyabinsk

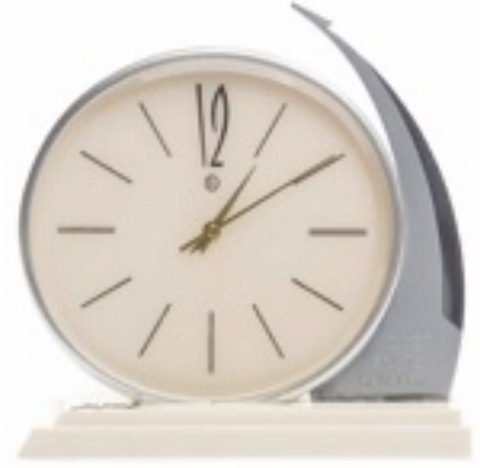

Fuente: Museo Estatal de Historia de los Urales del Sur.

Las futuras generaciones eran el objetivo del partido para relanzar el socialismo, y por ello, los motivos infantiles se alineaban al discurso orientado al futuro (Rüthers, 2011: 210). De acuerdo con Rüthers, el vínculo entre esas dos temáticas se observó en varios niveles, lo que fomentaba que los pequeños soñaran con ser cosmonautas: literatura infantil, paredes de escuelas, juguetes, parques infantiles, envoltorio de productos destinados a los niños, entre otros.
Figura 12. Vostok Lata de caramelos. (1960s).

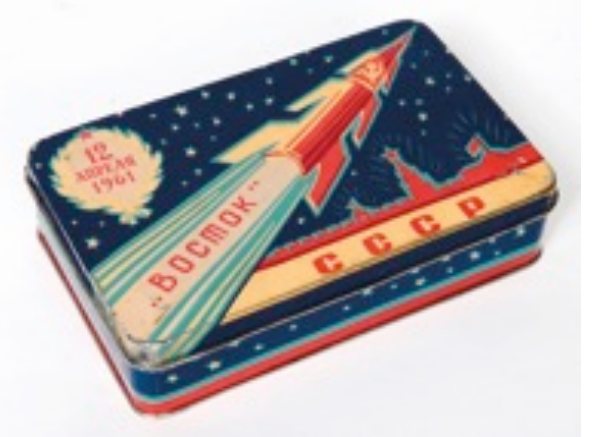

Fuente: Museo del Diseño de Moscú.

Figura 13. Parque infantil soviético con una recreación del Vostok 3

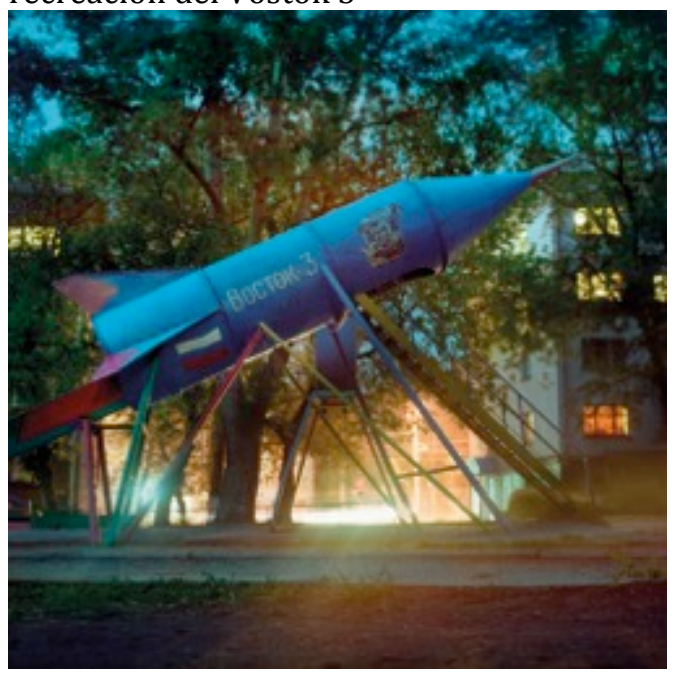

Fuente: Foto de Ivan Mikhailov, "The Calvet Journal", 2015.

Existen muchos ejemplos de diseño gráfico inspirados en la exploración del hombre en el espacio: en carteles, postales, sellos, entre otros. El ámbito gráfico es un capítulo aparte en la historia del diseño soviético. Heredero del constructivismo, fue un ejemplo sólido de la expresividad del diseño de los artistas soviéticos y no dependía de la precaria industria del país (Karpova, 2013) (A. Voronina, correo electrónico, 15 de mayo de 2019). La presencia constante de los colores azul y rojo, además de representar respectivamente, el cosmos y el comunismo, también hacía referencia al estilo constructivista. En la temática solían mezclarse símbolos del futuro y del espacio, con los típicos iconos del comunismo, pautados en imágenes de trabajadores, obreros y agricultores. 
Figura 14. "A través de los Mundos y de los Siglos" (1965). Cartel para concienciación social. Diseño de: Miron Lukyanov y Vasily Ostrovski.

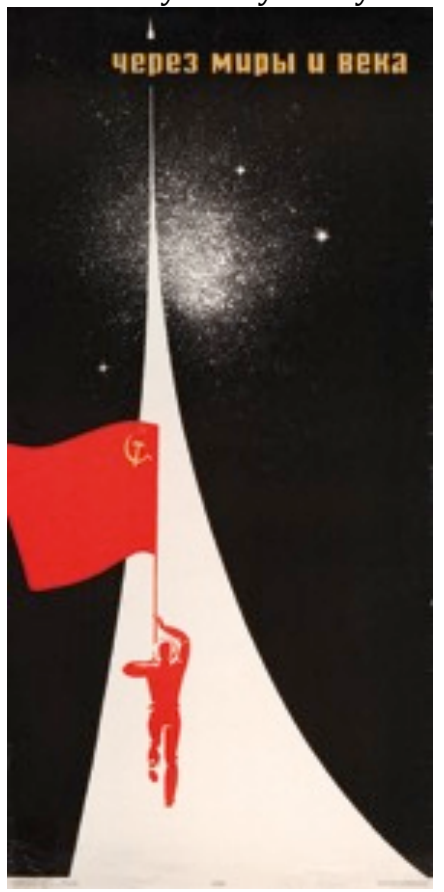

Fuente: Museo del Diseño de Moscú.

Figura 15. "Gloria al pueblo soviético, pioneros en el espacio!”. (1962). Cartel para concienciación social. Diseño de: V.Valikov

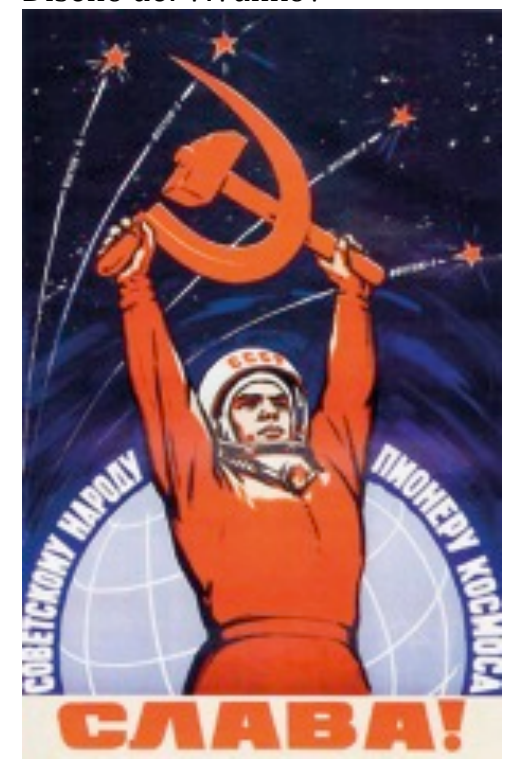

Fuente: Russia Beyond.

\section{El fin del optimismo por el espacio}

El entusiasmo de los soviéticos por el espacio empezó a apagarse a finales de los años sesenta, debido a una serie de acontecimientos sociales, económicos y políticos, que despertaron sentimientos de nostalgia e incertidumbre, además de hacerles cuestionar la legitimidad del Partido Comunista en el poder (Siddiqi, 2011: 295).

La muerte de Sergey Korolyov -diseñador jefe del programa espacial- en 1966, y posteriormente, de Yuri Gagarin en 1968, hicieron que el pueblo se sumiera en una profunda tristeza. Los detalles que salieron a la luz sobre los años de Korolyov como prisionero en el periodo estalinista, así como las circunstancias misteriosas alrededor del accidente de avión de Gagarin, provocaron que la población sospechara del cinismo del gobierno (Idem: 295-296) (Gerovitch, 2015: 151).

Había un gran abismo entre todo el capital invertido en el desarrollo de la tecnología militar y espacial y la vida de la población en la posguerra. La mala calidad y la escasez de alimentos, indignaba a los ciudadanos respecto a la gran inversión de dinero en los programas espaciales, en detrimento de la inversión de recursos para la vida cotidiana $y$, el estancamiento económico en la URSS a finales de los sesenta, hizo mella en el entusiasmo por la era espacial (Siddiqi, 2011: 295).

El golpe definitivo a la confianza de la URSS en su futuro como potencia tecnológica y científica, llegó a través del aterrizaje del hombre estadounidense en la Luna en 1969, que demostró que la Unión Soviética ya no estaba en la vanguardia de la carrera espacial. Al igual que en 1957 occidente había sido sacudido por el "shock Sputnik", la bandera de EEUU clavada en la superficie lunar avivó las dudas del pueblo sobre el futuro que se les había prometido (Idem: 296). La "nostalgia por el futuro" fue la expresión que dio nombre al sentimiento melancólico de los soviéticos respecto a la promesa fallida de que el socialismo y el cosmos traerían un gran futuro al país: 'lo que pudo ser, pero que nunca se materializó' (A. Voronina, correo electrónico, 15 de mayo de 2019) (Siddiqi, 2011: 299). A partir de ahí, la temática cósmica del entorno visual y material, empezó a generar sentimientos ambiguos: lo que anteriormente representaba alegría y optimismo por el futuro, pasó a personificar tristeza, decepción e hipocresía. 


\section{Discusiones y Conclusiones}

El diseño soviético de la década de 60 , tanto para uso doméstico como público, admite una serie de interpretaciones, pero, sobre todo, deja claro que el entusiasmo por el cosmos estaba reflejado en él. Tanto una estrategia del Partido Comunista, como un sentimiento social genuino, el diseño espacial representó el "zeitgeist" -el espíritu social del pueblo soviético de esos años-, por lo que simboliza las ambigüedades encontradas en la sociedad del período estudiado.

A principios de la era espacial, el diseño encarnó la expansión y la libertad de una nueva Era. Aquellos que vivían en un régimen totalitario, vigilados y privados de sus libertades, soñaban y empezaban a expresarse y a conocer nuevas realidades, a la vez que iniciaban un proceso de demanda de bienes de calidad. En forma de pequeños deseos mundanos $y$ capitalistas, el diseño empezó a ser una amenaza al proyecto socialista. Como afirmó Azrikan, "el diseño en aquella época fue (...) un agujero en el telón de acero"19. Dentro de ese análisis, la falta de diseño en el país, fue significativa para la disolución de la URSS.

Posiblemente, los colores alegres y los mensajes optimistas impresos en esos diseños no fueran solamente un mensaje de los dirigentes, sino que también emitían una nota subversiva de los ingenieros-artísticos (diseñadores), que a través de su trabajo, prometían un estilo de vida mejor. Lejos de ser implementados en el país, esos proyectos de mejora del entorno social, según los autores consultados, quedarían como una propuesta "utópica ${ }^{20}$ ", imposibilitados por la precariedad de la industria o por la interferencia ideológica del Partido, lo que acentuó la desilusión de los artistas y de los ciudadanos.

Una vez más, el diseño se reafirma como parte significativa de la cultura material y visual. A través suyo se reflejaron los deseos de un pueblo -y de un sistema-, y fue una expresión simbólica de una época. A través del análisis de esos artefactos se encuentra la "utopía" presente en la vida de los soviéticos. Asimismo, la interpretación del diseño cósmico deja al

\footnotetext{
${ }^{19}$ Véase Design in the USSR (2018: 234)

${ }^{20}$ Diseño utópico es una expresión para referirse a los diseños de los ingenieros artísticos de la institución VNIITE que no pasaban del papel (N. del autor).
}

descubierto que la semiología no depende sólo de las intenciones del emisor del mensaje (Partido Comunista y diseñadores), sino principalmente de quienes lo descifraban: el pueblo. Aunque la intención inicial había sido crear una Propaganda para relanzar el socialismo, según crecía la decepción social por el régimen, el diseño cósmico pasó de personificar el optimismo por un gran proyecto de país, a evocar significados menos alentadores -como el engaño y la hipocresía. Desde esa perspectiva, se podría afirmar que, según evolucionaba la sociedad, cambiaba la interpretación sobre su medio material y visual.

Se sugiere que este importante capítulo de la historia del diseño mundial de la década de sesenta, sea incorporado a las demás narrativas occidentales de la historia de la disciplina. Además de los relatos, por ejemplo, sobre la Escuela HfG Ulm o el surgimiento del Movimiento de Métodos en Diseño (DMM) entre otros, se debe añadir, acaso, la historia de la era espacial soviética, que incluye la Fundación del VNIITE, la mayor institución de diseño del mundo, poco conocida y estudiada en los relatos occidentales.

El análisis de los artefactos de la época estudiada, amplía las posibilidades de poner en debate las funciones del diseño dentro de la sociedad. Difundir la existencia de un sistema tan complejo de diseño en un país de economía planificada, con objetos proyectados para una larga duración, pone en cuestión la idea de que el diseño subsiste solamente gracias a los preceptos capitalistas e industriales, lo que da lugar a discusiones sobre la sostenibilidad, cuestión apremiante dentro de la disciplina a día de hoy.

La temática espacial sigue siendo un poderoso vehículo de discurso político y social. Sesenta años después, el gobierno actual aún utiliza los iconos que suscitan el orgullo en la población rusa, como herramienta política en la construcción de la nueva identidad rusa, según el curso político "patriótico" de Vladimir Putin. Por otra parte, se observó que la sociedad civil también rescata esos símbolos espaciales, como vehículo de discurso para la reconstrucción de su memoria cultural. 
Se pudo comprobar, tras la búsqueda de referencias 21 sobre el diseño soviético del periodo estudiado, que existen muy pocos estudios teóricos dedicados al tema. Entre ellos pueden destacarse: Design in the USSR: 19501989 (2018), VNIITE: Discovering Utopia - Lost Archives of Soviet Design (2018) y Unsong Icons of Soviet Design (2011), y de la década de 1990s, VNIITE, Dinosaur of Totalitarianism or Plato's Academy of Design? (1999). Este estudio podría servir de punto de partida para la exploración de otros temas relacionados, como son el diseño en la cultura en sociedades totalitarias, el diseño en una economía planificada, y el diseño sostenible.

\section{Agradecimientos}

En el presente trabajo de investigación, las autoras expresan su gratitud a Aleksandra Sankova y a Anastasia Voronina, por las entrevistas concedidas, respectivamente, en nombre del Museo del Diseño de Moscú y del Museo Estatal de Historia de los Urales del Sur.

${ }^{21}$ Se realizaron búsquedas relacionadas con el tema en cuatro idiomas: en ruso, inglés, portugués y español. 
URSS: Optimismo por el espacio en el diseño de los años 60

\section{Referencias}

Aleksiévich, S. (2015). El Fin del Homo Sovieticus. Barcelona: Acantilado.

Azrikan, D. (1999). VNIITE, Dinosaur of Totalitarianism or Plato's Academy of Design? Design Issues, 15(3), 45-77. DOI: 10.2307/1511884. https://www.jstor.org/stable/1511884

Bayazit, N. (2004). Investigating Design: A Review of Forty Years of Design Research. Design Issues, 20, 1629. DOI: https://doi.org/10.1162/074793604772933739

Cardoso, E.C.S y Santos, J.M. (2019). Utopia no fim do homem soviético. Intexto, 44, 236-254. DOI: http://dx.doi.org/10.19132/1807-8583201944.236-254

Central Intelligence Agency (CIA) (24 de julio de 1959). Transcription Kitchen Debate. U.S. Embassy, Moscow, Soviet Union. Recuperado de https://www.cia.gov/library/readingroom/docs/1959-07-24.pdf

Cropley, D. H., Cropley, A. J. \& Sandwith, B. L. (2017). Creativity in the Engineering Domain. In J. Kaufman, V. P. Glaveanu, and J. Baer (Eds.), The Cambridge Handbook of Creativity Across Domains, Chapter 15, 261-275. New York: Cambridge University Press.

Devezas, T. et al. (2012). The struggle for space: Past and future of the space race. Technological Forecasting \& Social Change, 79, 963-985. https://doi.org/10.1016/j.techfore.2011.12.006

Fry, T. (1989). A Geography of Power: Design History and Marginality. Design Issues, 6(1), Design in Asia and Australia, 15-30. https://www.jstor.org/stable/1511575

Gerovitch, S. (2015). Soviet Space Mythologies: Public Images, Private Memories, and the Making of a Cultural Identity. Pennsylvania: University of Pittsburgh Press.

Idov, M. (Ed.) (2011). Made in Russia: Unsung Icons of Soviet Design. New York: Rizzoli International Publications.

Iron Curtain. Encyclopaedia Britannica [version electrónica]. NewYork, EU: Encyclopaedia Britannica Inc., www.britannica.com/event/Iron-Curtain

Karpova, J. (2013). Accommodating 'design': introducing the Western concept into Soviet art theory in the 1950s - 60s. European Review of History: Revue européenne d'histoire, 20(4), 627-647, https://doi.org/10.1080/13507486.2012.763160

Langrish, J.Z. (2016). The Design Methods Movement: From Optimism to Darwinism. Proceedings of DRS 2016, Design Research Society 50th Anniversary Conference. Brighton, UK, 27-30 June 2016 https://www.drs2016.org/222

Malashenko, U. (02 de abril de 2019). The First Group of Female Cosmonauts Were Trained to Conquer the Final Frontier. Smithsonian Magazine. Recuperado de https://www.smithsonianmag.com/science-nature/first-group-female-cosmonauts-trainedconquer-final-frontier-180971900/

Margolin, V. (2005). A World History of Design and the History of the World. Journal of Design History, 18(3), 235-243. https://doi.org/10.1093/jdh/epi043

Margolin, V. (2009). Design in History. Design Issues, 25(2), 94-105. https://www.mitpressjournals.org/doi/pdf/10.1162/desi.2009.25.2.94

Maurer, E., Richers, J., Rüthers, M. y Scheide, C. (Eds.) (2011). Soviet Space Culture: Cosmic Enthusiasm in Socialist Societies. London: Palgrave Macmillan.

Moscow Design Museum (2018). Designed In The USSR. 1950 - 1989. London: Phaidon Press.

Powaski, R. (2000). La Guerra Fría. Estados Unidos y la Unión Soviética, 1917-1991. Barcelona: Editorial Crítica.

Redacción BBC News (29 de julio de 2018). El excepcional debate entre Jruschov y Nixon que tuvo lugar en una cocina en plena Guerra Fría. BBC. https://www.bbc.com/mundo/noticias-44986980 Recuperado el 05 de marzo de 2019.

Russia Beyond. History. (12 de abril de 2018). How did posters make people proud of Soviet success in space exploration? (Pics). Russia Beyond. Recuperado de https://www.rbth.com/history/328012-sovietspace-posters-make-proud. Recuperado el 15 de enero de 2020. 
Rospopina, S. (15 de septiembre de 2015). How space exploration replaced religion in the USSR. The Guardian. https://www.theguardian.com/world/2015/sep/17/ussr-space-exploration-religionrussia. Recuperado el 21 de mayo de 2019.

Sankova, A. y Druzhinina, O. (2018). VNIITE: Discovering Utopia - Lost Archives of Soviet Design. London: Unit Editions.

Siddiq, A. (2011) From Cosmic Enthusiasm to Nostalgia for the Future: A Tale of Soviet Space Culture. In Soviet Space Culture: Cosmic Enthusiasm in Socialist Societies. Maurer, E., Richers, J., Rüthers, M., and Scheide, C. (Eds.), 283-306. London: Palgrave Macmillan.

Turkina, O. (2014). Soviet Space Dogs. London: Fuel.

Woodham, J. (2004). A Dictionary of Modern Design. New York: Oxford University Press.

Woodham, J. (2005). Local, National and Global: Redrawing the Design Historical Map. Journal of Design History, 18(3), 257-267. http://www.jstor.org/stable/3527286

\section{Documentales:}

“Мода на космос"(Moda espacial). Burkova, E. (productora). Digovtsev, M. (director) (2016). Сделано в CCCР" (Hecho en la URSS) [serie de televisión]. Rusia. Productora: Мир 24.

«История российского дизайна» (La historia del diseño ruso). Sankova, A. у Chirkov, S. (autoras) Chirkov, S. (guionista) (2018). Rusia. Productora: Moscow Design Museum.

\section{Web:}

Moscow Design Museum. http://moscowdesignmuseum.ru

Museo Estatal de Historia de los Urales del Sur. http://chelmuseum.ru/english_ver

Astronaut.http://astronaut.ru/ 